\title{
Alpha Power Extended Inverse Weibull Poisson Distribution: Properties, Inference, and Applications to lifetime data
}

\author{
Jemilohun Vincent Gbenga ${ }^{1}$, Ipinyomi Reuben Adeyemi ${ }^{1}$ \\ ${ }^{1}$ Afebabalola University Ado-Ekiti, Department of Statistics, University of Ilorin, Kwara State, Nigeria \\ Correspondence: Jemilohun Vincent Gbenga, Afebabalola University, Ado-Ekiti, Ekiti, Nigeria
}

Received: September 13, 2021

Accepted: November 26, $2021 \quad$ Online Published: December 21, 2021

doi:10.5539/ijsp.v11n1p10

URL: https://doi.org/10.5539/ijsp.v11n1p10

\begin{abstract}
In this paper, a new four-parameter extended inverse Weibull distribution called Alpha power Extended Inverse Weibull Poisson distribution is introduced using the alpha power Poisson generator. This method adds two shape parameters to a baseline distribution thereby increasing its flexibility and applicability in modeling lifetime data. We study the structural properties of the new distribution such as the mean, variance, quantile function, median, ordinary and incomplete moments, reliability analysis, Lorenz and Bonferroni curves, Renyi entropy, mean waiting time, mean residual life, and order statistics. We use the method of maximum likelihood technique for estimating the model parameters of Alpha power extended inverse Weibull distribution and the corresponding confidence intervals are obtained. The simulation method is carried out to evaluate the performance of the maximum likelihood estimate in terms of their Absolute Bias and Mean Square Error using simulated data. Two lifetime data sets are presented to demonstrate the applicability of the new model and it is found that the new model has superior modeling power when compare to Inverse Weibull distribution, Alpha Power Poisson inverse exponential distribution, Alpha Power Extended Inverse Weibull distribution, and Alpha Power Extended Inverse Exponential distribution.
\end{abstract}

Keywords: reliability analysis, Lorenz and Bonferroni and curves, order statistics, moments, maximum likelihood estimation

\section{Introduction}

Adding an extra shape parameter to a classical (conventional) distribution is very common in statistical distribution theory. Often introducing an extra parameter(s) brings more flexibility to a class of distribution functions essentially for data analysis purposes to improve the modeling potential of the classical distribution. For example, Azzalini (1985) introduced the skew-normal distribution by introducing an extra parameter to the normal distribution to induce more flexibility into the normal distribution. Mudholkar and Srivastava (1993) proposed a method that introduced an extra parameter to a two-parameter Weibull distribution and called it exponentiated Weibull model which has two shape parameters and one scale parameter. Marshall and Olkin (1997) introduced another method that adds a parameter to any distribution function; two special cases were considered namely when $\mathrm{X}$ follows exponential or Weibull distribution and derived many properties of this proposed model. The well-known generators are the following: the beta-G family of distribution which was developed and studied by Eugene et al. (2002), Cordeiro and de Castro (2011) developed the Kumaraswamy-G family of distribution, exponentiated generalized-G family of distribution was proposed and studied by Cordeiro et al. (2013), Nofal et al. (2017) developed the generalized transmuted-G family of distribution, transmuted exponentiated generalized-G family of distribution was proposed and studied by Yousof et al. (2015), transmuted geometric-G family of distribution was developed and studied by Afify et al. (2016), Kumaraswamy transmuted-G family of distribution was studied by Afify et al. (2016b). Alizadeh et al. (2017) developed the generalized odd generalized exponential family of distribution, exponentiated Weibull-H family of distribution was proposed and developed by Cordeiro et al. (2017), exponentiated generalized-G Poisson family of distribution was developed and studied by Aryal and Yousof (2017), Alizadeh et al. (2018) proposed and studied transmuted Weibull-G family of distribution, Marshall-Olkin generalized-G Poisson family of distribution was developed and studied by Korkmaz et al. (2018). Oluyede, et al. (2018) introduced the gamma Weibull-G family of distributions by combining the gamma generator with the Weibull-G family of distributions which was defined by Bourguignon et al. (2014) and odd Lomax-G family of distribution was studied by Cordeiro et al. (2019) Recently, the alpha power transformation was proposed and studied by Mahdavi and Kundu (2017).

Let $\bar{H}$ be the CDF of any continuous random variable X, then the CDF of Alpha Power Transformed (APT) family is 
given by

$$
G(x)=\left\{\begin{array}{cc}
\frac{\alpha^{\bar{H}(x)}-1}{\alpha-1}, & \text { if } \alpha>0, \alpha \neq 1 \\
\bar{H}(x), & \alpha=0
\end{array}\right.
$$

And the associated pdf is given by

$$
g(x)=\left\{\begin{array}{cl}
\frac{\log \alpha}{\alpha-1} \bar{h}(x) \alpha^{\bar{H}(x)}, & \text { if } \alpha>0, \alpha \neq 1 \\
\bar{H}(x), & \text { if } \alpha=0
\end{array}\right.
$$

The transformation has been used by several researchers to obtain alpha transformed distributions. Namely, Dey et al. (2017a, 2017b, 2018, 2019) studied the properties of the new extensions of generalized exponential distribution with an application to ozone data, a new extension of Weibull distribution with application to real-life data, extended Weibull distribution with application to real-life data, alpha transformed inverse Lindley distribution which exhibits upside-down bathtub shape failure rate, and alpha power transformed Lindley distribution with applications to earthquake data. Hassan et al. (2018) investigate the properties of alpha power transformed extended exponential distribution, alpha power Weibull distribution was studied by Nasser et al. (2017). Ogunde et al. (2020a, 2020b) studied the properties of alpha power extended Bur II distribution and alpha power extended inverted Weibull distribution respectively.

Motivated by the advantages offered by a generalized distribution which makes it more relevant in modeling lifetime data that are non-monotonic, exhibiting different shapes of the hazard function ranges from increasing, decreasing, and bathtub shapes, as well as the versatility of compounding alpha power Inverse Weibull and Poisson distribution in modeling real-life data. We study a new generalization called the Alpha power extended Inverse Weibull Poisson (APEIWP) distribution which possesses these properties.

We are also motivated to study the APEIWP distribution because of its simplicity and extensive usage of IW distribution in modeling lifetime events. Also, the current generalization provides a wider application even to complex situations that involve different shapes of the hazard function.

\section{The Model, Sub-Models, and Properties of Alpha Power Extended Inverse Weibull Poisson (APEIWP) Distribution}

The probability density function (PDF) and the associated distribution function (CDF) of the two-parameter inverse Weibull (IW) distribution is given by

$$
\bar{h}(x ; \eta, \omega)=\eta \omega x^{-\omega-1} e^{-\eta x^{-\omega-1}}, \quad x>0
$$

and

$$
\bar{H}(x ; \eta, \omega)=e^{-\eta x^{-\omega}}, \quad x>0
$$

where $\eta$ is a positive scale parameter $(\eta>0)$ and $\omega$ is a positive shape parameter $(\omega>0)$, respectively. Keller et al. (1982) used the IW distribution to describe the wear and tear phenomena of some mechanical components such as crankshaft and pistons of diesel engines. In addition, the IW model has many important applications in Insurance, reliability engineering, useful life, wear-out periods, service records, and life testing, see Khan and King (2012).

Several generalizations of the Inverse Weibull distribution have been proposed and studied, see, for example, beta Inverse Weibull by Khan (2010), generalized Inverse Weibull was studied by de Gusmao et al. (2011), modified Inverse Weibull by Khan and King (2012), Pararai et al. (2014) studied the properties of gamma Inverse Weibull, Kumaraswamy generalized Inverse Weibull by Oluyede and Yang (2014), Aryal and Elbatal (2015 ) investigated the properties of Kumaraswamy modified Inverse Weibull distribution, the properties of Marshall-Olkin Inverse Weibull was investigated by Okasha et al. (2017), alpha power Inverse Weibull was studied by Basheer (2019), and the extended Inverse Weibull distribution was developed and studied by Said Alkarni et al. (2020).

Given that $\bar{H}(x)$ is the CDF of a distribution given in (4), then inserting (4) in (1) given another distribution called Alpha power extended Inverse Weibull distribution (APEIW) which CDF is given by

$$
G(x)=\left\{\begin{array}{cc}
\frac{\alpha^{e^{-\eta x^{-\omega}}}-1}{\alpha-1}, & \text { if } \alpha>0, \alpha \neq 1 \\
\alpha^{e^{-\eta x^{-\omega}}}, & \alpha=0
\end{array}\right.
$$

And the corresponding PDF is given by 


$$
g(x)=\left\{\begin{array}{cl}
\frac{\log \alpha}{\alpha-1} \eta \omega x^{-\omega-1} e^{-\eta x^{-\omega}} \alpha^{-\eta x^{-\omega}} & \text { if } \alpha,>0, \alpha \neq 1 \\
\eta \omega x^{-\omega-1} e^{-\eta x^{-\omega}}, & \text { if } \alpha=0
\end{array}\right.
$$

Suppose that $\mathrm{X}$ has at the Alpha power extended Inverse Weibull distribution where its PDF and CDF are given in (5) and (6) respectively. Given $N$, let $X_{1}, \ldots, X_{N}$ be independent and identically distributed random variables from APEIW distribution. Let $\mathrm{N}$ be distributed according to the zero truncated Poisson distribution with pdf

$$
P(N=n)=\frac{\lambda^{n} e^{-\lambda}}{n !\left(1-e^{-\lambda}\right)}, \quad n=1,2,3, \ldots, \lambda>0
$$

Let $X=\max \left(Z_{1}, \ldots, Z_{N}\right)$, then the CDF of $X / N=n$ is given by

$$
F_{X / N=n}(x)=\left(\frac{\alpha^{e^{-\eta x^{-\omega}}}-1}{\alpha-1}\right)^{n},
$$

Which is the exponentiated alpha power extended Inverse Weibull distribution. The Alpha power Extended Inverse Weibull Poisson distribution is the marginal CDF of $X$, given by

$$
F(x ; \eta, \omega, \lambda)=\frac{1-\exp \left[-\lambda\left(\frac{\alpha^{e^{-\eta x^{-\omega}}}-1}{\alpha-1}\right)\right]}{e^{-\lambda}-1}, \quad x>0
$$

Where $\omega$, and $\lambda$ are positive shape parameters and $\eta$ is a positive scale parameter respectively. The corresponding $A P E I W P$ density function is given by

$$
f(x)=\frac{\eta \omega \lambda x^{-\omega-1} \log \alpha e^{-\eta x^{-\omega}} \alpha^{e^{-\eta x^{-\omega}}} \exp \left[-\lambda\left(\frac{\alpha^{e^{-\eta x^{-\omega}}}-1}{\alpha-1}\right)\right]}{(\alpha-1)\left(e^{-\lambda}-1\right)}, x>0
$$

Where $\omega$, and $\lambda$ are positive shape parameters and $\eta$ is a positive scale parameter respectively. The graph of the CDF and PDF are respectively drawn below in figure (1) and (2) for various values of the parameters of APEIWP distribution.

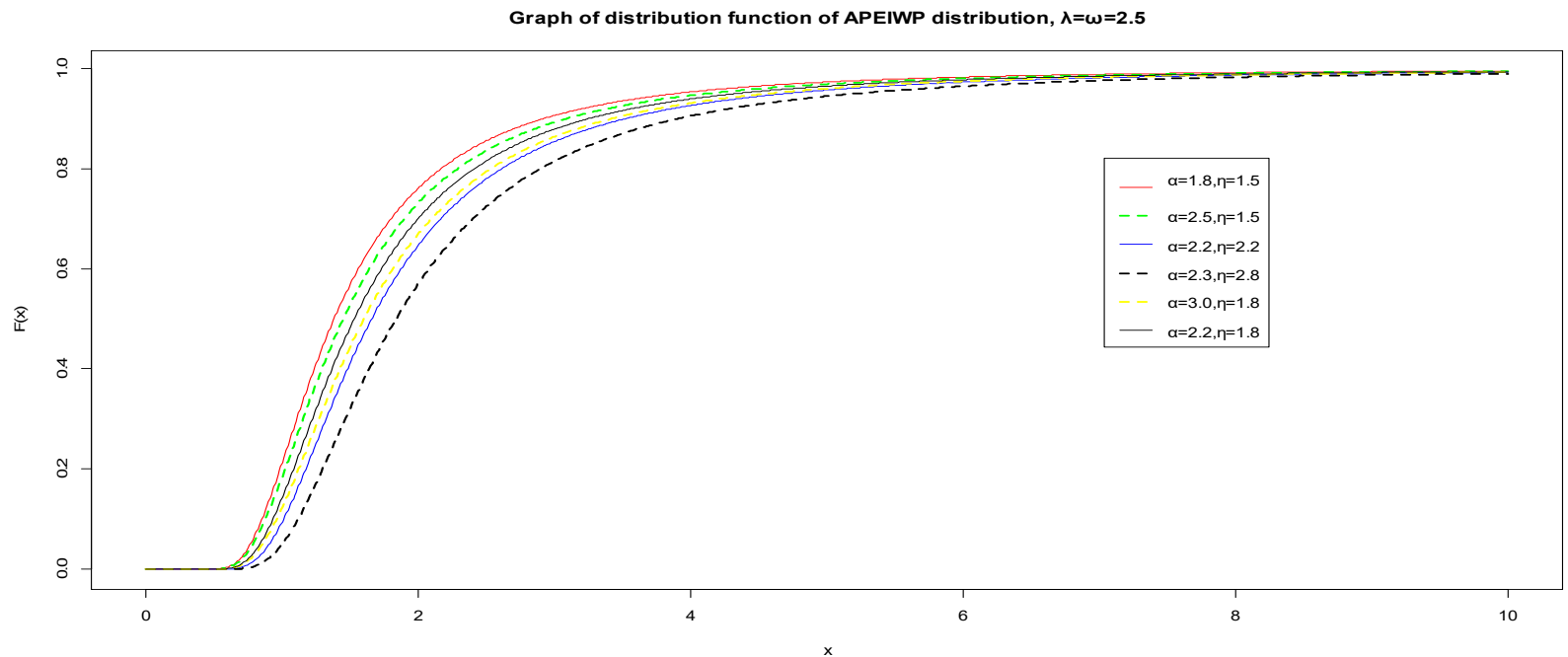

Figure 1. The graph of the CDF of APEIWP distribution

$\checkmark$ Figure 1 indicates that the APEIWP distribution has a proper density function. 


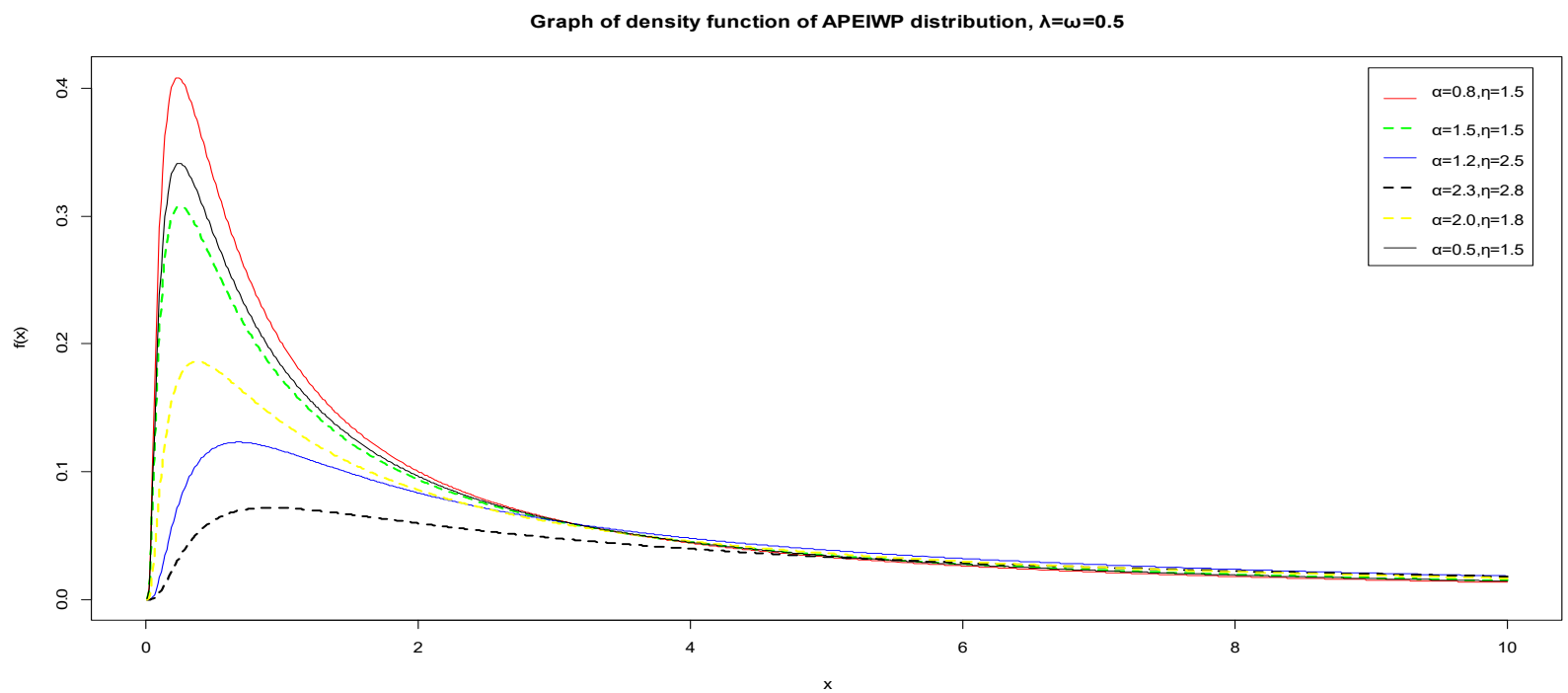

Figure 2. The graph of the PDF of APEIWP distribution

$\checkmark \quad$ Figure 2 indicates that the graph of APEIWP distribution is unimodal

The survival function $(S(x))$ is obtained by using the relation,

$$
\begin{gathered}
S(x)=1-F(x) \\
=1-\frac{1-\exp \left[-\lambda\left(\frac{\lambda \alpha^{e^{-\eta x^{-\omega}}}-1}{\alpha-1}\right)\right]}{e^{-\lambda}-1}
\end{gathered}
$$

And the hazard function is given as

$$
\begin{gathered}
h(x)=\frac{f(x)}{S(x)} \\
=\frac{\eta \omega \lambda x^{-\omega-1} \log \alpha e^{-\eta x^{-\omega}} \alpha^{e^{-x^{-\omega}}} \exp \left[-\lambda\left(\frac{\lambda \alpha^{e^{-\eta x^{-\omega}}}-1}{\alpha-1}\right)\right]}{(\alpha-1)\left(e^{-\lambda}-1\right)\left(1-\frac{1-\exp \left[-\lambda\left(\frac{\lambda \alpha^{e^{-\eta x^{-\omega}}}-1}{\alpha-1}\right)\right]}{e^{-\lambda}-1}\right)}
\end{gathered}
$$

Figures 3 and 4 are the graph of the hazard function of APEIWP distribution for various values of the parameters. The graph shows that the hazard function of APEIWP model exhibits the non-monotone failure rate or upside-down bathtub failure rate for the values of the parameters considered. 


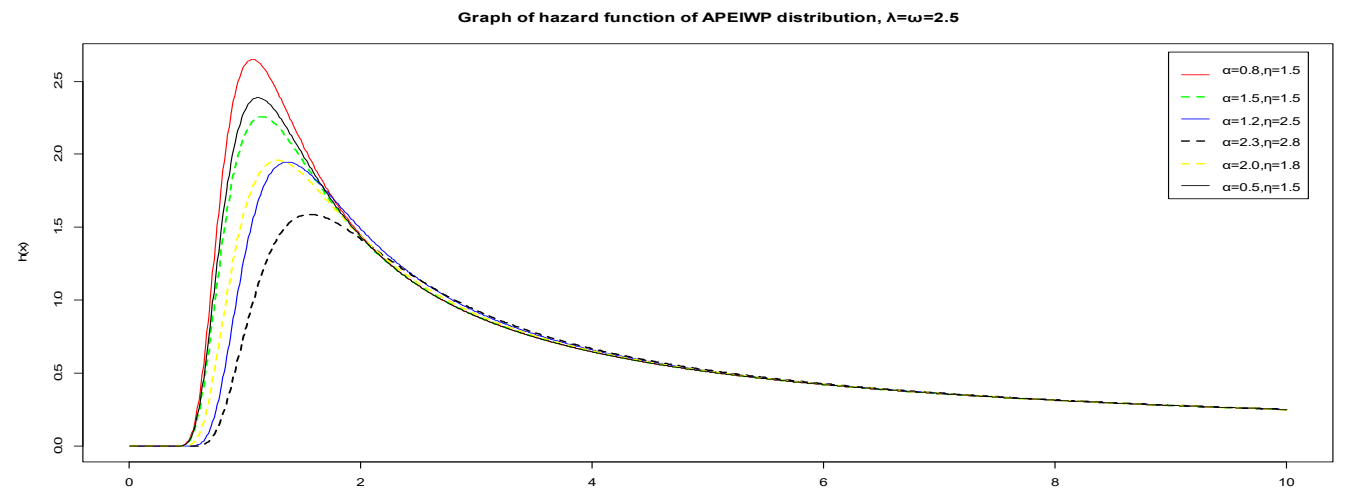

Figure 3. The graph of the hazard function of APEIWP distribution

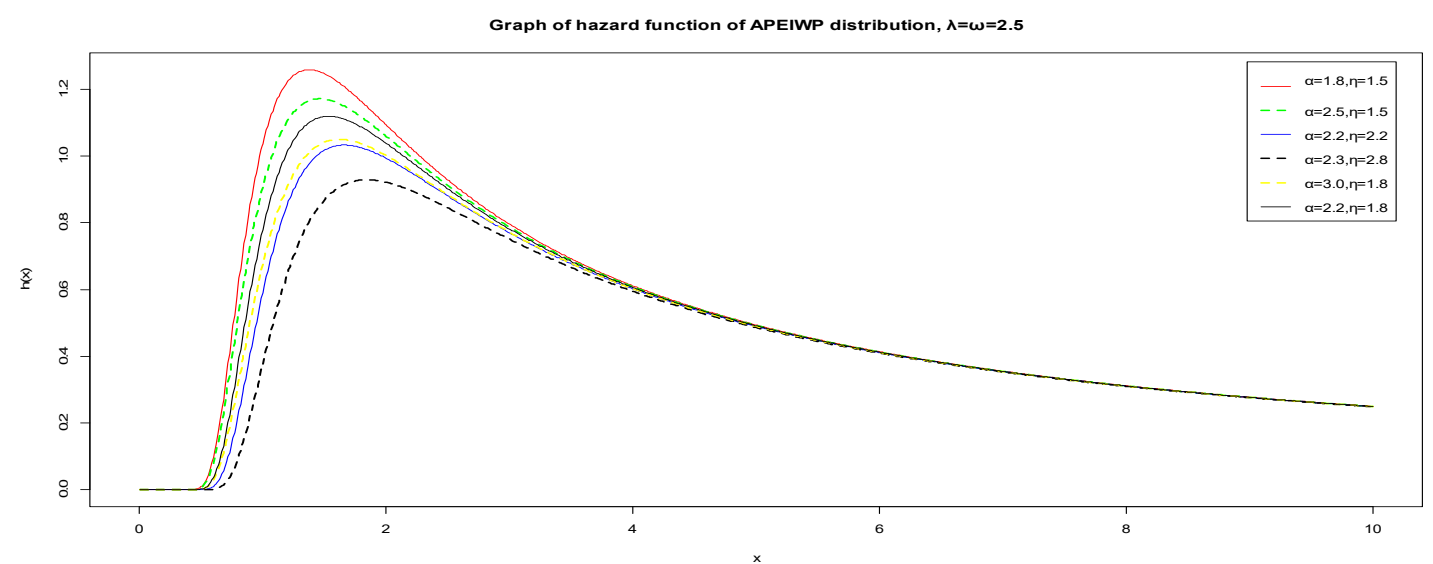

Figure 4. The graph of the hazard function of APEIWP distribution

\subsection{Quantile Function}

Quantile function can be defined as an inverse of the distribution function. Consider the relation

$$
F(X)=U \Rightarrow X=F^{-1}(U)
$$

Where $U$ follows standard Uniform distribution. The $p^{\text {th }}$ quantile of APEIWP distribution is given by

$$
X_{u}=\left[-\frac{1}{\eta}\left\{\left(\frac{1}{\log \alpha}\right) \log \left[(\alpha-1)\left(1-\frac{1}{\lambda} \log \left[(\alpha-1)\left\{1-u\left(e^{-\lambda}-1\right)\right\}\right]\right)\right]\right\}\right]^{-1 / \omega}
$$

The lower quartile, mean, and the upper quartile APEIWP distribution can be obtained from (11) by setting the value of $u$ to be $0.25,0.5$, and 0.75 respectively. An expression for the lower quartile, median, and upper quartile is given as

$$
\begin{aligned}
X_{0.25} & =\left[-\frac{1}{\eta}\left\{\left(\frac{1}{\log \alpha}\right) \log \left[(\alpha-1)\left(1-\frac{1}{\lambda} \log \left[(\alpha-1)\left\{1-0.25\left(e^{-\lambda}-1\right)\right\}\right]\right)\right]\right\}\right]^{-1 / \omega} \\
X_{0.5} & =\left[-\frac{1}{\eta}\left\{\left(\frac{1}{\log \alpha}\right) \log \left[(\alpha-1)\left(1-\frac{1}{\lambda} \log \left[(\alpha-1)\left\{1-0.5\left(e^{-\lambda}-1\right)\right\}\right]\right)\right]\right\}\right]^{-1 / \omega}
\end{aligned}
$$

and

$$
X_{0.75}=\left[-\frac{1}{\eta}\left\{\left(\frac{1}{\log \alpha}\right) \log \left[(\alpha-1)\left(1-\frac{1}{\lambda} \log \left[(\alpha-1)\left\{1-0.75\left(e^{-\lambda}-1\right)\right\}\right]\right)\right]\right\}\right]^{-1 / \omega}
$$

\section{Random numbers generation}

Random numbers can be generated for the $\operatorname{APEIWP}(\alpha, \lambda, \eta, \omega)$ distribution, for this let, simulating values of random variable $\mathrm{X}$ with the $\mathrm{CDF}$ given in (7) and $q$ denote a uniform random variable in $(0,1)$, then the simulated values of $\mathrm{X}$ are obtained by as, 


$$
X=\left[-\frac{1}{\eta}\left\{\left(\frac{1}{\log \alpha}\right) \log \left[(\alpha-1)\left(1-\frac{1}{\lambda} \log \left[(\alpha-1)\left\{1-q\left(e^{-\lambda}-1\right)\right\}\right]\right)\right]\right\}\right]^{-1 / \omega}
$$

\section{Skewness and Kurtosis}

The quantile function can be used to determine Bowley's skewness $\left(B_{s}\right)$, Kenny and keeping (1992) and Moor's kurtosis $\left(M_{k}\right)$, Moor's (1988). These measures are obtained as

$$
B_{S}=\frac{Q\left(\frac{3}{4}\right)+Q\left(\frac{1}{4}\right)-2 Q\left(\frac{2}{4}\right)}{Q\left(\frac{3}{4}\right)-Q\left(\frac{1}{4}\right)}
$$

and

$$
M_{k}=\frac{Q\left(\frac{7}{8}\right)-Q\left(\frac{5}{8}\right)+Q\left(\frac{3}{8}\right)-Q\left(\frac{1}{8}\right)}{Q\left(\frac{6}{8}\right)-Q\left(\frac{2}{8}\right)}
$$

Table 1 drawn below gives the values of Bowley's skewness $\left(B_{s}\right)$ and kurtosis $\left(M_{k}\right)$ for various values of the parameters of APEIW distributions taken $\eta=1.3$ and $\omega=1.2$.

\begin{tabular}{|c|c|c|c|c|}
\hline & $\alpha=2, \lambda=0.25$ & $\alpha=1.8, \lambda=5.2$ & $\alpha=1.8, \lambda=4.2$ & $\alpha=2, \lambda=3.2$ \\
\hline$Q\left(\frac{1}{4}\right)$ & 2.9862 & 3.0184 & 3.0817 & 8.4553 \\
\hline$Q\left(\frac{2}{4}\right)$ & 1.5227 & 2.4677 & 2.4073 & 5.0001 \\
\hline$Q\left(\frac{3}{4}\right)$ & 0.9554 & 2.2449 & 2.1499 & 3.7314 \\
\hline$Q\left(\frac{1}{8}\right)$ & 5.5389 & 3.0184 & 3.0817 & 14.6247 \\
\hline$Q\left(\frac{3}{8}\right)$ & 2.0364 & 2.6128 & 2.5794 & 6.1975 \\
\hline$Q\left(\frac{5}{8}\right)$ & 1.1922 & 2.3471 & 2.2669 & 4.2501 \\
\hline$Q\left(\frac{7}{8}\right)$ & 0.7716 & 2.1569 & 2.0404 & 3.3493 \\
\hline$B_{S}$ & -0.4413 & -0.4239 & -0.4475 & -0.4628 \\
\hline$M_{k}$ & 1.9318 & 0.7703 & 0.7821 & 1.9746 \\
\hline
\end{tabular}

\subsection{Mixture Representation for the Density Function}

The mixture representation of the density function is a very useful tool used in deriving the statistical properties of generalized distribution. In this section, the mixture representation of the APEIWP density function is obtained. Using the following series representation:

$$
\begin{gathered}
e^{y}=\sum_{t=0}^{\infty} \frac{y^{t}}{t !} \\
(1-z)^{y}=\sum_{t=0}^{\infty}(-1)^{t}\left(\begin{array}{l}
y \\
t
\end{array}\right) z^{t}
\end{gathered}
$$




$$
\alpha^{v}=\sum_{t=0}^{\infty} \frac{(\log \alpha)^{t}}{t !} v^{t}
$$

Using the series expansion given in (15) in (8), we have

Also, using (16) in (18), we have

$$
\exp \left[-\lambda\left(\frac{\lambda \alpha^{e^{-\eta x^{-\omega}}}-1}{\alpha-1}\right)\right]=\sum_{i=0}^{\infty} \frac{(-\lambda)^{i}}{i !}\left\{\left(\frac{\alpha^{e^{-\eta x^{-\omega}}}-1}{\alpha-1}\right)\right\}^{i}
$$

$$
\begin{gathered}
\left\{\left(\frac{\alpha^{e^{-\eta x^{-\omega}}}-1}{\alpha-1}\right)\right\}^{i}=(-1)^{i}\left(\frac{1}{\alpha-1}\right)^{i}\left(1-\alpha^{e^{-\eta x^{-\omega}}}\right)^{i} \\
=(-1)^{i}\left(\frac{1}{\alpha-1}\right)^{i} \sum_{j=0}^{\infty}\left(\begin{array}{l}
i \\
j
\end{array}\right)(-1)^{j}\left(\alpha^{e^{-\eta x^{-\omega}}}\right)^{j}
\end{gathered}
$$

Consequently,

$$
f(x)=\frac{\eta \omega \lambda x^{-\omega-1} \log \alpha e^{-\eta x^{-\omega}}}{(\alpha-1)\left(e^{-\lambda}-1\right)}\left(\frac{1}{\alpha-1}\right)^{i} \sum_{i, j=0}^{\infty}\left(\begin{array}{l}
i \\
j
\end{array}\right)(-1)^{i+j} \frac{(-\lambda)^{i}}{i !}\left(\frac{1}{\alpha-1}\right)^{i} \alpha^{(1+j) e^{-\eta x^{-\omega}}}
$$

applying (17) to (19), finally we have,

$$
f(x)=\frac{\eta \omega \lambda}{\left(e^{-\lambda}-1\right)} \sum_{i, j, k=0}^{\infty}\left(\begin{array}{l}
i \\
j
\end{array}\right)(-1)^{i+j} \frac{(-\lambda)^{i}}{i !}\left(\frac{1}{\alpha-1}\right)^{i+1}(1+j)^{k}(\log \alpha)^{k} x^{-\omega-1} e^{-(1+k) \eta x^{-\omega}}
$$

The above expression is a density of inverse Weibull distribution with scale parameter $(1+k) \eta$ and shape parameter $\alpha$

\section{Ordinary and Incomplete Moment}

The ordinary moments of distribution play a very important role in statistical applications. The $r^{\text {th }}$ moment of a random variable $\mathrm{X}$ can be obtained using

$$
E\left(X^{r}\right)=\mu_{r}^{\prime}=\int_{-\infty}^{\infty} x^{r} f(x) d x
$$

Putting (20) in (21), we have

$$
\mu_{r}^{\prime}=\frac{\eta \omega \lambda}{\left(e^{-\lambda}-1\right)} \sum_{i, j, k}^{\infty}\left(\begin{array}{l}
i \\
j
\end{array}\right)(-1)^{i+j} \frac{(-\lambda)^{i}}{i !}\left(\frac{1}{\alpha-1}\right)^{i+1}(1+j)^{k}(\log \alpha)^{k} f^{\eta, \omega}
$$

where

$$
f^{\eta, \omega}=\int_{-\infty}^{\infty} x^{r-\omega-1} e^{-(1+k) \eta x^{-\omega}} d x
$$

By letting $z=(1+k) \eta x^{-\omega}, x=z^{-\frac{1}{\omega}}((1+k) \eta)^{\frac{1}{\omega}}$ and putting it in (22), we have

$$
f^{\eta, \omega}=\frac{1}{\omega}((1+k) \eta)^{\frac{r}{\omega}} \Gamma(1-r / \omega)
$$

Finally $r^{\text {th }}$ moment of APEIWP distribution is given by 


$$
\mu_{r}^{\prime}=\frac{\eta \lambda}{\left(e^{-\lambda}-1\right)} \sum_{i, j, k}^{\infty} M_{i, j, k}^{\lambda}((1+k) \eta)^{\frac{r}{\omega}} \Gamma(1-r / \omega)
$$

where

$$
M_{i, j, k}^{\lambda}=\left(\begin{array}{l}
i \\
j
\end{array}\right)(-1)^{i+j} \frac{(-\lambda)^{i}}{i !}\left(\frac{1}{\alpha-1}\right)^{i+1}(1+j)^{k}(\log \alpha)^{k}
$$

$r<\omega$. Fo $r=1,2, \ldots \Gamma($.$) is the gamma function. By taking r=1$, we obtain the mean of $X$ that is, $\mu_{1}^{\prime}=\mu$. The variance of $\mathrm{X}$ obtained by $\sigma^{2}=E\left[(X-\mu)^{2}\right]=\mu_{2}^{\prime}-\mu^{2}$. Also, we can determine the $r^{\text {th }}$ central moment and $r^{\text {th }}$ cumulant of $X$ respectively defined by

$$
\mu_{r}=E\left[(X-\mu)^{r}\right]=\sum_{m=0}^{r}\left(\begin{array}{l}
r \\
m
\end{array}\right) \mu_{r-m}^{\prime}(-1)^{m} \mu^{m}, \quad k_{r}=\mu_{r}^{\prime}-\sum_{m=1}^{r-1}\left(\begin{array}{c}
r-1 \\
m-1
\end{array}\right) k_{m} \mu_{r-m}^{\prime},
$$

Taking $k=\mu$, several measures of skewness and kurtosis based on the central moments (or cumulants) can be obtained.

Table 1 drawn below gives the first six moments and variance $\left(\sigma^{2}\right)$ and coefficient of variation $(C V)$ of $A P E I W P$ distributions. The values for $C V=\frac{\sigma}{\mu}=\sqrt[2]{\frac{\mu_{2}^{\prime}}{\mu^{2}}-1}$

Table 2. First six moments and $\sigma^{2}$ and $C V$ for $A P E I W P$ distribution

\begin{tabular}{|c|c|c|c|c|}
\hline Moment & $\alpha=0.2, \lambda=0.5$ & $\alpha=0.5, \lambda=1.0$ & $\alpha=1.5, \lambda=2.5$ & $\alpha=5.5, \lambda=5.5$ \\
\hline$\mu_{1}^{\prime}$ & 1.0664 & 1.0867 & 1.0696 & 1.0364 \\
\hline$\mu_{2}^{\prime}$ & 1.1769 & 1.2268 & 1.1809 & 1.0917 \\
\hline$\mu_{3}^{\prime}$ & 1.3645 & 1.4623 & 1.3637 & 1.1733 \\
\hline$\mu_{4}^{\prime}$ & 1.7140 & 1.9029 & 1.6940 & 1.2978 \\
\hline$\mu_{5}^{\prime}$ & 2.5349 & 2.9491 & 2.4452 & 1.5175 \\
\hline$\mu_{6}^{\prime}$ & 6.5953 & 8.1991 & 6.0485 & 2.2605 \\
\hline$\sigma^{2}$ & 0.0397 & 0.0459 & 0.0369 & 0.0176 \\
\hline$C V$ & 0.1868 & 0.1972 & 0.1796 & 0.1280 \\
\hline
\end{tabular}

An expression for an Incomplete moment is given by

$$
\varphi_{r}(t)=\int_{0}^{t} x^{r} f(x) d x
$$

Putting (20) in (24), we have

$$
\boldsymbol{\varphi}_{r}(\boldsymbol{t})=\frac{\eta \omega \lambda}{\left(e^{-\lambda}-1\right)} \sum_{-\infty}^{\infty}\left(\begin{array}{l}
i \\
j
\end{array}\right)(-1)^{i+j}(-1)^{i+j} \frac{(-\lambda)^{i}}{i !}\left(\frac{1}{\alpha-1}\right)^{i+1}(1+j)^{k}(\log \alpha)^{k} f^{*}
$$

where

$$
f^{*}=\int_{0}^{t} x^{r-\omega-1} e^{-(1+k) \eta x^{-\omega}} d x
$$

Also, by letting $z=(1+k) \eta x^{-\omega}, x=z^{-\frac{1}{\omega}}((1+k) \eta)^{\frac{1}{\omega}}$ and putting it in (25), we have

$$
f^{\eta, \omega}=\frac{1}{\omega}((1+k) \eta)^{\frac{r}{\omega}} \Gamma\left(1-r / \omega,(1+k) \eta t^{-\omega}\right)
$$

Finally the $r^{\text {th }}$ incomplete moment of APEIWP distribution is given by 


$$
\varphi_{r}(t)=\frac{\eta \lambda}{\left(e^{-\lambda}-1\right)} \sum_{i, j, k}^{\infty} M_{i, j, k}^{\lambda}((1+k) \eta)^{\frac{r}{\omega}} \Gamma\left(1-r / \omega,(1+k) \eta t^{-\omega}\right)
$$

Where $M_{i, j, k}^{\lambda}$ is defined in (23), $\Gamma(m, n)=\int_{n}^{\infty} v^{m-1} e^{-v} d v$ is the complementary incomplete gamma function.. the first incomplete moment of APEIWP distribution is given as

$$
\varphi_{1}(t)=\frac{\eta \lambda}{\left(e^{-\lambda}-1\right)} \sum_{i, j, k}^{\infty} M_{i, j, k}^{\lambda}((1+k) \eta)^{\frac{1}{\omega}} \Gamma\left(1-1 / \omega,(1+k) \eta t^{-\omega}\right)
$$

The mean deviation, $\gamma_{1}(x)$ and median deviation, $\gamma_{2}(x)$, can be obtained by using the relation, $\gamma_{1}(x)=2 \mu F(\mu)-$ $2 \gamma_{1}(\mu)$ and $\gamma_{2}(x)=\mu-2 \gamma_{1}(M)$. Where $\mu=E(X)$ and $M$ is the median of the APEIWP random variable. Both the $\gamma_{1}(\mu)$ and $\gamma_{1}(M)$ are calculated from the first incomplete moment as given in (27)

\section{Inequality Measures}

Inequality measures can be applied in biomedical sciences, product quality control economics, insurance and demography, and many more. Here we consider the following inequality measures:

\subsection{Mean Residual Life (MRL)}

Residual life is defined as the expected additional life length for a unit that is alive at age $t$, and it is represented mathematically by $m_{x}(t)=E(X-t / X>t), t>0$.

The $M R L$ of $X$ can be obtained by using the formula:

$$
m_{x}(t)=\frac{\left[1-\varphi_{1}(t)\right]}{S(t)}-t
$$

Where $S(t)$ is the survival function of $X$ and $\varphi_{1}(t)$ as given in (27). Then we have

$$
m_{x}(t)=\frac{1}{s(t)}\left(\frac{\eta \lambda}{\left(e^{-\lambda}-1\right)} \sum_{i, j, k}^{\infty} M_{i, j, k}^{\lambda}((1+k) \eta)^{\frac{1}{\omega}} \Gamma\left(1-1 / \omega,(1+k) \eta t^{-\omega}\right)\right)-t,
$$

The mean inactivity time (MIT) (mean waiting time) is defined by $M_{x}(t)=E(t X / X \leq t), t>0$, and it can be obtained by the formula:

$$
M_{x}(t)=t-\left[\frac{\varphi_{1}(t)}{F(t)}\right]
$$

Also putting (27) in (30), we obtain an expression for MIT for APEIWP distribution as

$$
M_{x}(t)=t-\left[\frac{\frac{\eta \lambda}{\left(e^{-\lambda}-1\right)} \sum_{i, j, k}^{\infty} M_{i, j, k}^{\lambda}((1+k) \eta)^{\frac{1}{\omega}} \Gamma\left(1-1 / \omega,(1+k) \eta t^{-\omega}\right)}{F(t)}\right]
$$

\subsection{Bonferroni And Lorenz Curves}

The Bonferroni and Lorenz curve of APEIWP distribution are respectively given by

$$
\mathfrak{B}_{F}=\frac{1}{\mu F(t)} \int_{0}^{t} x^{r} f(x) d x
$$

Since,

$$
\int_{0}^{t} x^{r} f(x) d x=\frac{\eta \lambda}{\left(e^{-\lambda}-1\right)} \sum_{i, j, k}^{\infty} M_{i, j, k}^{\lambda}((1+k) \eta)^{\frac{1}{\omega}} \Gamma\left(1-1 / \omega,(1+k) \eta t^{-\omega}\right)
$$

therefore

$$
\mathfrak{B}_{F}(t)=\frac{1}{\mu F(t)} \frac{\eta \lambda}{\left(e^{-\lambda}-1\right)} \frac{\eta \lambda}{\left(e^{-\lambda}-1\right)} \sum_{i, j, k}^{\infty} M_{i, j, k}^{\lambda}((1+k) \eta)^{\frac{1}{\omega}} \Gamma\left(1-1 / \omega,(1+k) \eta t^{-\omega}\right)
$$

And the Lorenz curve

$$
L_{F}(t)=\frac{1}{\mu} \int_{0}^{t} x^{r} f(x) d x
$$




$$
=\frac{1}{\mu} \frac{\eta \lambda}{\left(e^{-\lambda}-1\right)} \sum_{i, j, k}^{\infty} M_{i, j, k}^{\lambda}((1+k) \eta)^{\frac{1}{\omega}} \Gamma\left(1-1 / \omega,(1+k) \eta t^{-\omega}\right)
$$

\subsection{Stress-Strenght Parameter}

Suppose $X_{1}$ and $X_{2}$ be two continuous and independent random variables where $X_{1} \sim \operatorname{APEIWP}\left(\alpha_{1}, \lambda_{1}, \eta, \omega\right)$ and $X_{2} \sim A P E I W P\left(\alpha_{2}, \lambda_{2}, \eta, \omega\right)$, then the stress-strength parameter, say, , is defined as

$$
f=\int_{-\infty}^{\infty} f_{1}(x) F_{2}(x) d x
$$

Using the CDF and the PDF of APEIWP in (35), the stress-strength parameters can be obtained as

$$
\begin{gathered}
f=\frac{F_{2}(x)}{e^{-\lambda_{1}-1}}-\frac{1}{e^{-\left(\lambda_{2}+\lambda_{1}+2\right)}} \sum_{i, j, l, m, p, q}^{\infty}(-1)^{i+l}\left(\begin{array}{l}
i \\
j
\end{array}\right)\left(\begin{array}{l}
l \\
m
\end{array}\right)\left(\alpha_{2}-1\right)^{i+1}\left(\log \alpha_{2}\right)^{j+1}\left(\log \alpha_{1}\right)^{p} \frac{i^{q} l^{p}}{i ! l ! p ! q !} \\
\times\left(\frac{1}{(q+1) \eta}\right)
\end{gathered}
$$

\section{Entropy}

The Renyi entropy of APEIWP distribution can be obtained using a formula suggested by Renyi (1961) as

$$
R_{v}=\frac{1}{v-1} \int_{-\infty}^{\infty} f^{v}(x) d x
$$

Inserting (8) in (36), we have

$$
R_{v}=\frac{1}{v-1} \int_{-\infty}^{\infty}\left\{\frac{\eta \omega \lambda x^{-\omega-1} \log \alpha e^{-\eta x^{-\omega}} \alpha^{e^{-x^{-\omega}}} \exp \left[-\lambda\left(\frac{\alpha^{e^{-\eta x^{-\omega}}}-1}{\alpha-1}\right)\right]}{(\alpha-1)\left(e^{-\lambda}-1\right)}\right\}^{v} d x
$$

Using Taylor series expansion in (15), (16), and (17), we have

$$
\int_{-\infty}^{\infty} f^{v}(x) d x=\frac{\eta^{v} \omega^{v-1} \lambda^{v}}{\left(e^{-\lambda}-1\right)^{v}} \sum_{i, j, k}^{\infty} \frac{(v \lambda)^{i}(v+j)^{k}(-1)^{i+j}}{(\alpha-1)^{v+i} i ! k !}\left(\begin{array}{l}
i \\
j
\end{array}\right) \int_{-\infty}^{\infty} x^{-v(\omega+1)} e^{-(k+v) \eta x^{-\omega}} d x
$$

By letting the value of $z=(k+v) \eta x^{-\omega}, x=z^{-1 / \omega}((k+v) \eta)^{-1 / \omega}$,

$$
\int_{-\infty}^{\infty} f^{v}(x) d x=\frac{\eta^{v} \omega^{v-1} \lambda^{v}}{\left(e^{-\lambda}-1\right)^{v}} \sum_{i, j, k}^{\infty} \frac{(v \lambda)^{i}(v+j)^{k}(-1)^{i+j}}{(\alpha-1)^{v+i} i ! k !}\left(\begin{array}{l}
i \\
j
\end{array}\right) G_{\omega}^{*}
$$

Where,

$$
G_{\omega}^{*}=((k+v) \eta)^{\frac{1-v(\omega+1)}{\omega}} \Gamma\left\{1+\frac{(\omega+1)(v-1)}{\omega}\right\}
$$

Finally, the Renyi entropy of APEIWP distribution is given by

$$
R_{v}=\frac{1}{v-1} \frac{\eta^{v} \omega^{v-1} \lambda^{v}}{\left(e^{-\lambda}-1\right)^{v}} \sum_{i, j, k}^{\infty} \frac{(v \lambda)^{i}(v+j)^{k}(-1)^{i+j}}{(\alpha-1)^{v+i} i ! k !}\left(\begin{array}{l}
i \\
j
\end{array}\right) G_{\omega}^{*}
$$

\subsection{Order Statistics}

Suppose a random sample is drawn from the $\operatorname{APEIWP}(\boldsymbol{\alpha}, \eta, \omega, \lambda)$ denoted by $\underline{X}$ of size $m$ have the following order statistics denoted by $X_{1: r}<X_{2: n}<\ldots<X_{r: n}$. Then, the PDF of the $r^{\text {th }}$ order statistics is given by 


$$
f_{r: n}(x)=\frac{n !}{(r-1) !(n-r) !} F^{r-1}(x)[1-F(x)]^{n-r} f(x)
$$

Using the series expansion (16) in (39), we have

$$
f_{r: n}(x)=\frac{n !}{(r-1) !(n-r) !} \sum_{j=0}^{n-r}(-1)^{j}\left(\begin{array}{c}
n-r \\
j
\end{array}\right) F^{j+r-1}(x) f(x)
$$

Considering, $F^{j+r-1}(x) f(x)$ and further applying the Taylor series given in (15) and (16), we have

$$
\begin{gathered}
F^{j+r-1}(x) f(x)=\frac{\eta \omega \lambda}{\left(e^{-\lambda}-1\right)^{j+r}} x^{-\omega-1} \sum_{l, m, p, q=0}^{\infty} \frac{(-1)^{l+m+p}}{m ! q !(\alpha-1)^{m+1}}(-\lambda(l+1))^{m}\left(\begin{array}{c}
m \\
p
\end{array}\right)(\log \alpha)^{1+q} \\
\times\left(\begin{array}{c}
j+r-1 \\
l
\end{array}\right)(p+1)^{q} e^{-(q+1) \eta x^{-\omega}}
\end{gathered}
$$

Finally the $r^{\text {th }}$ order statistics of APEIWP distribution is given by

$$
\begin{gathered}
f_{r: n}(x)=\frac{n ! \eta \omega \lambda}{(r-1) !(n-r) !} \sum_{j=0}^{\infty} \sum_{l, m, p, q=0}^{\infty} \frac{(-1)^{j+\_l+m+p}}{m ! q !(\alpha-1)^{m+1}\left(e^{-\lambda}-1\right)^{j+r}}(-\lambda(l+1))^{m}\left(\begin{array}{c}
n-r \\
j
\end{array}\right) \\
\times\left(\begin{array}{c}
m \\
p
\end{array}\right)\left(\begin{array}{c}
j+r-1 \\
l
\end{array}\right)(\log \alpha)^{1+q}(p+1)^{q} e^{-(q+1) \eta x^{-\omega}}
\end{gathered}
$$

\section{Simulation Study}

In this section, we carry out t6he simulation study to ascertain the performance of MLEs of APEIWP distribution. The

\begin{tabular}{|c|c|c|c|c|c|c|}
\hline & parameter & mean & $A B$ & $S D$ & $\delta^{2}$ & MSE \\
\hline \multirow{4}{*}{$n=50$} & $\alpha$ & 0.3226 & 1.6774 & 0.4206 & 0.1769 & 2.9906 \\
\hline & $\lambda$ & 0.9420 & 0.4420 & 1.7967 & 3.2281 & 3.4235 \\
\hline & $\eta$ & 3.5929 & 2.2929 & 0.8280 & 0.6856 & 5.9430 \\
\hline & $\omega$ & 1.4026 & 0.2026 & 0.3593 & 0.1291 & 0.1702 \\
\hline \multirow{4}{*}{$n=100$} & $\alpha$ & 0.2933 & 1.7067 & 0.2964 & 0.0879 & 3.0007 \\
\hline & $\lambda$ & 0.7827 & 0.2827 & 1.0925 & 1.1936 & 1.2735 \\
\hline & $\eta$ & 3.1679 & 1.8679 & 0.5142 & 0.2647 & 3.7538 \\
\hline & $\omega$ & 1.3456 & 0.01456 & 0.2220 & 0.0493 & 0.0705 \\
\hline \multirow{4}{*}{$n=200$} & $\alpha$ & 0.4834 & 1.5166 & 0.4982 & 0.2482 & 2.5483 \\
\hline & $\lambda$ & 1.0536 & 0.5536 & 0.9687 & 0.9384 & 1.2449 \\
\hline & $\eta$ & 3.1661 & 1.8661 & 0.4276 & 0.1828 & 3.6651 \\
\hline & $\omega$ & 1.2840 & 0.0840 & 0.1964 & 0.0386 & 0.0457 \\
\hline \multirow{4}{*}{$n=300$} & $\alpha$ & 0.4490 & 1.5510 & 0.1409 & 0.0196 & 2.4252 \\
\hline & $\lambda$ & 0.7217 & 0.5783 & 0.3242 & 0.1051 & 0.4395 \\
\hline & $\eta$ & 2.8109 & 1.5109 & 0.1583 & 0.0251 & 2.3079 \\
\hline & $\omega$ & 1.2667 & 0.0667 & 0.0743 & 0.0055 & 0.0099 \\
\hline \multirow{4}{*}{$n=400$} & $\alpha$ & 0.4707 & 1.5293 & 0.2466 & 0.0608 & 2.3996 \\
\hline & $\lambda$ & 0.8501 & 0.3501 & 0.5583 & 0.3117 & 0.4342 \\
\hline & $\eta$ & 2.9522 & 1.6522 & 0.2672 & 0.0714 & 2.8012 \\
\hline & $\omega$ & 1.2558 & 0.0558 & 0.1235 & 0.0153 & 0.0184 \\
\hline \multirow{4}{*}{$n=500$} & $\alpha$ & 0.4917 & 1.5083 & 0.2611 & 0.0682 & 2.3432 \\
\hline & $\lambda$ & 0.8693 & 0.3693 & 0.4945 & 0.2445 & 0.3809 \\
\hline & $\eta$ & 2.9023 & 1.6023 & 0.2397 & 0.0575 & 2.6248 \\
\hline & $\omega$ & 1.2402 & 0.0402 & 0.1092 & 0.0119 & 0.0135 \\
\hline
\end{tabular}
random number generation is obtained with its quantile function (qf) given in (11). We generated $\mathrm{N}=1000$ sample sizes 50, 100, 200, 300, 400, and 500 from APEIWP distribution using its qf taking $\alpha=2.0, \lambda=0.5, \eta=1.3$ and $\omega=1.2$. Then we calculated the empirical means, standard deviation (SD), variance $\left(\sigma^{2}\right)$ absolute bias $(\mathrm{AB})$. We observed that as the sample size increases, for each of the parameter estimates the mean square error approaches zero as expected.

Table 2. The empirical means, $A B, S D, \sigma^{2}$ and $M S E$ for $A P E I W P$ distribution parameters 


\subsection{Maximum Likelihood Estimation}

Let $X_{1}, X_{2}, \ldots, X_{n}$ be a random sample drawn from APEIWP $(\alpha, \eta, \lambda, \omega)$ then the likelihood function is given by

$$
\boldsymbol{L}(\underline{\boldsymbol{x}}, \alpha, \eta, \lambda, \omega)=\prod_{\boldsymbol{i}=\mathbf{0}}^{\boldsymbol{n}} \frac{\eta \omega \lambda x^{-\omega-1} \log \alpha e^{-\eta x^{-\omega}} \alpha^{e^{-\eta x^{-\omega}}} \exp \left[-\lambda\left(\frac{\alpha^{e^{-\eta x^{-\omega}}}-1}{\alpha-1}\right)\right]}{(\alpha-1)\left(e^{-\lambda}-1\right)},
$$

Then, taking $z^{x_{i}}=e^{-\eta x^{-\omega}}$ the $\log$ likelihood function $(\log L=l)$ is given by

$$
\begin{array}{r}
l=n \log \left(\frac{\eta \omega \lambda}{(\alpha-1)\left(e^{-\lambda}-1\right)}\right)+n \log [\log (\alpha)]-\eta \sum_{i=1}^{n} x_{i}^{-\omega}+\log (\alpha) \sum_{i=1}^{n} z^{x_{i}} \\
-\frac{\lambda}{(\alpha-1)} \sum_{i=1}^{n}\left(\alpha^{z^{x_{i}}}-1\right)
\end{array}
$$

We differentiate (45) with respect $\alpha, \lambda, \eta$ and $\omega$, to obtain the element of the score vector $\left(V_{\alpha}=\frac{\partial l}{\partial \alpha}, V_{\lambda}=\frac{\partial l}{\partial \lambda}, V_{\eta}=\right.$ $\left.\frac{\partial l}{\partial \eta}, V_{\omega}=\frac{\partial l}{\partial \omega}\right)^{T}$. The elements of the score vector are given by

$V_{\alpha}=\frac{n}{\alpha-1}+\frac{n(\alpha-1-\alpha \log (\alpha)}{\alpha(\alpha-1) \log (\alpha)}+\frac{1}{\alpha} \sum_{i=1}^{n} z^{x_{i}}-\frac{\lambda}{(\alpha-1)^{2}} \sum_{i=1}^{n} \alpha^{z^{x_{i}}}+\frac{\lambda}{\alpha(\alpha-1)} \sum_{i=1}^{n} \alpha^{z^{x_{i}}} z^{x_{i}}$

$V_{\lambda}=\frac{n}{\lambda}-\frac{n e^{-\lambda}}{e^{-\lambda}-1}-\frac{1}{(\alpha-1)} \sum_{i=1}^{n}\left(\alpha^{z^{x_{i}}}-1\right)$,

$V_{\eta}=\frac{n}{\eta}-\sum_{i=1}^{n} x_{i}{ }^{-\omega}-\log (\alpha) \sum_{i=1}^{n} x_{i}^{-\omega} z^{x_{i}}-\frac{\lambda}{(\alpha-1)} \log (\alpha) \sum_{i=1}^{n} x_{i}^{-\omega} \alpha^{z^{x_{i}}} z^{x_{i}}$

$V_{\omega}=\frac{n}{\omega}-\eta \sum_{i=1}^{n} x_{i}^{-\omega} \log (\alpha)-\eta \log (\alpha) \sum_{i=1}^{n} x_{i}^{-\omega} e^{-\eta x^{-\omega}}-\eta \operatorname{og}(\alpha) \sum_{i=1}^{n} x_{i}^{-\omega} \log (x) z^{x_{i}} \alpha^{z^{x_{i}}}$

By setting the non-linear system of equations $V_{\alpha}=V_{\lambda}=V_{\eta}=V_{\omega}=0$ and obtaining a feasible solution by solving the simultaneously, the MLE of the parameters of the APEIWP model are obtained. However, these equations cannot be solved analytically, statistical software can be employed to solve them numerically by using iterative methods such as Newton-Raphson algorithms. To carry out interval estimation of the model parameters, we require the observed information matrix

$$
H(\xi)=-\left[\begin{array}{llll}
V_{\alpha \alpha} & V_{\alpha \lambda} & V_{\alpha \eta} & V_{\alpha \omega} \\
V_{\lambda \alpha} & V_{\lambda \lambda} & V_{\lambda \eta} & V_{\lambda \omega} \\
V_{\eta \alpha} & V_{\eta \lambda} & V_{\eta \eta} & V_{\eta \omega} \\
V_{\omega \alpha} & V_{\omega \lambda} & V_{\omega \eta} & V_{\omega \omega}
\end{array}\right]
$$

Under certain standard regularity conditions as $n \rightarrow \infty$, the distribution of $\hat{\xi}$ can be approximated by a multivariate normal $N_{4}\left(0, H(\hat{\xi})^{-1}\right)$ distribution to construct approximate confidence intervals for the parameters. Here, $H(\hat{\xi})$ represent the total observed information matrix calculated at $\hat{\xi}$.

Asymptotic $(1-p) 100 \%$ confidence intervals for parameters can be obtained as

$$
\hat{\alpha} \pm Z p_{/ 2} \sqrt{\Sigma_{11}}, \quad \hat{\lambda} \pm Z p_{/ 2} \sqrt{\Sigma_{11}}, \quad \hat{\eta} \pm Z p_{/ 2} \sqrt{\Sigma_{11}}, \quad \widehat{\omega} \pm Z p_{/ 2} \sqrt{\Sigma_{11}}
$$

\subsection{Least Square Method (LSE)}

Let $x_{1}, \ldots, x_{n}$ be a random sample from APEIWP distribution with parameters $\alpha, \lambda, \eta$, and $\omega$. By considering the corresponding order statistics $X_{1: n}, \ldots, X_{n: n}$, taking $E\left[F\left(X_{i: n}\right)\right]=\frac{I}{n+1}$. The least square estimates can be obtained by 
minimizing the following expression

$$
L^{s}(\xi)=\sum_{i=1}^{n}\left[F\left(x_{i}\right)-E\left[F\left(x_{i}\right)\right]\right]^{2}=\sum_{i=1}^{n}\left[F\left(x_{i}\right)-\frac{i}{n+1}\right]^{2} .
$$

Minimizing $L^{s}(\xi)$ with respect to $\alpha, \lambda, \eta$ and $\omega$, we have the following system of non-linear equations:

$$
\begin{aligned}
& \frac{\partial L^{s}(\xi)}{\partial \alpha}=2 \sum_{i=1}^{n}\left[F\left(x_{i}\right)-\frac{i}{n+1}\right] F^{\prime}\left(x_{i}\right)_{\alpha}=0 \\
& \frac{\partial L^{s}(\xi)}{\partial \lambda}=2 \sum_{i=1}^{n}\left[F\left(x_{i}\right)-\frac{i}{n+1}\right] F^{\prime}\left(x_{i}\right)_{\lambda}=0 \\
& \frac{\partial L^{s}(\xi)}{\partial \eta}=2 \sum_{i=1}^{n}\left[F\left(x_{i}\right)-\frac{i}{n+1}\right] F^{\prime}\left(x_{i}\right)_{\eta}=0 \\
& \frac{\partial L^{s}(\xi)}{\partial \omega}=2 \sum_{i=1}^{n}\left[F\left(x_{i}\right)-\frac{i}{n+1}\right] F^{\prime}\left(x_{i}\right)_{\omega}=0
\end{aligned}
$$

Where $F^{\prime}\left(x_{i}\right)_{\alpha}=\frac{\partial y}{\partial \alpha} F\left(x_{i}\right), F^{\prime}\left(x_{i}\right)_{\lambda}=\frac{\partial y}{\partial \lambda} F\left(x_{i}\right) \quad F^{\prime}\left(x_{i}\right)_{\eta}=\frac{\partial y}{\partial \eta} F\left(x_{i}\right)$ and $F^{\prime}\left(x_{i}\right)_{\omega}=\frac{\partial y}{\partial \omega} F\left(x_{i}\right)$. These equations can be solved numerically by any software to obtain the estimates $\hat{\alpha}_{L S E}, \hat{\lambda}_{L S E}, \hat{\eta}_{L S E}$, and $\widehat{\omega}_{L S E}$

6.3 Weighted Least Square (WLS)

Let $x_{1}, \ldots, x_{n}$ be a random sample from APEIWP distribution with parameters $\alpha, \lambda, \eta$, and $\omega$. The likelihood function for a weighted least square estimate is given by

$$
\boldsymbol{W}(\xi)=\sum_{i=1}^{n} \frac{(n+1)^{2}(n+2)}{i(n-i+1)}\left[F\left(x_{i}\right)-\frac{i}{n+1}\right]^{2} .
$$

Minimizing $W(\xi)$ with respect to $\alpha, \lambda, \eta$ and $\omega$, we have the following system of non-linear equations:

$$
\begin{aligned}
& \frac{\partial W(\xi)}{\partial \alpha}=2 \sum_{i=1}^{n} \frac{(n+1)^{2}(n+2)}{i(n-i+1)}\left[F\left(x_{i}\right)-\frac{i}{n+1}\right] F^{\prime}\left(x_{i}\right)_{\alpha}=0 \\
& \frac{\partial W(\xi)}{\partial \lambda}=2 \sum_{i=1}^{n} \frac{(n+1)^{2}(n+2)}{i(n-i+1)}\left[F\left(x_{i}\right)-\frac{i}{n+1}\right] F^{\prime}\left(x_{i}\right)_{\lambda}=0 \\
& \frac{\partial W(\xi)}{\partial \eta}=2 \sum_{i=1}^{n} \frac{(n+1)^{2}(n+2)}{i(n-i+1)}\left[F\left(x_{i}\right)-\frac{i}{n+1}\right] F^{\prime}\left(x_{i}\right)_{\eta}=0 \\
& \frac{\partial W(\xi)}{\partial \omega}=2 \sum_{i=1}^{n} \frac{(n+1)^{2}(n+2)}{i(n-i+1)}\left[F\left(x_{i}\right)-\frac{i}{n+1}\right] F^{\prime}\left(x_{i}\right)_{\omega}=0
\end{aligned}
$$

This system of non-linear equations can be solved numerically by any software to obtain the estimates $\hat{\alpha}_{L S E}, \hat{\lambda}_{L S E}$, $\hat{\eta}_{L S E}$, and $\widehat{\omega}_{L S E}$

6.4 Cramer Von Mises (CVM)

Crammer von Mises is a type of minimum distance estimators. Let $x_{1}, \ldots, x_{n}$ be a random sample from APEIWP 
distribution with parameters $\alpha, \lambda, \eta$, and $\omega$. The likelihood function for Crammer von Mises estimate is given by

$$
C(\xi)=\frac{1}{12 n}+\sum_{i=1}^{n}\left[F\left(x_{i}\right)-\frac{2 i-1}{2 n}\right]^{2} .
$$

Minimizing $C(\xi)$ with respect to $\alpha, \lambda, \eta$ and $\omega$, we have the following system of non-linear equations:

$$
\begin{aligned}
& \frac{\partial C(\xi)}{\partial \alpha}=2 \sum_{i=1}^{n}\left[F\left(x_{i}\right)-\frac{2 i-1}{2 n}\right] F^{\prime}\left(x_{i}\right)_{\alpha}=0, \\
& \frac{\partial W(\xi)}{\partial \lambda}=2 \sum_{i=1}^{n}\left[F\left(x_{i}\right)-\frac{2 i-1}{2 n}\right] F^{\prime}\left(x_{i}\right)_{\lambda}=0 \\
& \frac{\partial W(\xi)}{\partial \eta}=2 \sum_{i=1}^{n}\left[F\left(x_{i}\right)-\frac{2 i-1}{2 n}\right] F^{\prime}\left(x_{i}\right)_{\eta}=0 \\
& \frac{\partial L^{s}(\xi)}{\partial \omega}=2 \sum_{i=1}^{n}\left[F\left(x_{i}\right)-\frac{2 i-1}{2 n}\right] F^{\prime}\left(x_{i}\right)_{\omega}=0
\end{aligned}
$$

These equations can be solved numerically by any software to obtain the estimates $\hat{\alpha}_{L S E}, \hat{\lambda}_{L S E}, \hat{\eta}_{L S E}$, and $\widehat{\omega}_{L S E}$ 6.5 Practical Applications

In this subsection, we evaluate the performance of the APEIWP distributions with the other four competing models to two reliability data sats. The data sets are described as follows:

The data set (data set 1). The data set was presented by Murthy et al. (2004) on the failure times (in weeks) of 50 components. The data set are: $0.013,0.065,0.111,0.111,0.163,0.309,0.426,0.535,0.684,0.747,0.997,1.284,1.304$, $1.647,1.829,2.336,2.838,3.269,3.977,3.981,4.520,4.789,4.849,5.202,5.291,5.349,5.911,6.018,6.427,6.456$, $6.572,7.023,7.087,7.291,7.787,8.596,9.388,10.261,10.713,11.658,13.006,13.388,13.842,17.152,17.283,19.418$, $23.471,24.777,32.795,48.105$.

The data set (data set 2). The data set is made up of failure time in hours of Kevlar 49/epoxy strands with pressure at $90 \%$ and was already studied by Andrews and Herzberg (2012). The data consists of 101 observations and the numbers are: $0.01,0.01,0.02,0.02,0.02,0.03,0.03,0.04,0.05,0.06,0.07,0.07,0.08,0.09,0.09,0.10,0.10,0.11,0.11,0.12,0.13$, $0.18,0.19,0.20,0.23,0.24,0.24,0.29,0.34,0.35,0.36,0.38,0.40,0.42,0.43,0.52,0.54,0.56,0.60,0.60,0.63,0.65$, $0.67,0.68,0.72,0.72,0.72,0.73,0.79,0.79,0.80,0.80,0.83,0.85,0.90,0.92,0.95,0.99,1.00,1.01,1.02,1.03,1.05$, $1.10,1.10,1.11,1.15,1.18,1.20,1.29,1.31,1.33,1.34,1.40,1.43,1.45,1.50,1.51,1.52,1.53,1.54,1.54,1.55$, $1.58,1.60,1.63,1.64,1.80,1.80,1.81,2.02,2.05,2.14,2.17,2.33,3.03,3.03,3.34,4.20,4.69,7.89$.

The descriptive statistics of the two data sets are given in Table 3 and the graph of Total Time on Test plot is given in figure 5 and Boxplot in figure 6. From this table, it can be observed that the two data sets are over-dispersed, leptokurtic, and positively skewed. Also, from figure 5, it can be observed that data 1 exhibits decreasing failure rate and data 2 exhibit a non-monotone failure rate

Table 3. Exploratory data Analysis of Failure data

\begin{tabular}{|c|c|c|}
\hline Discriptive statistics & Data 1 & Data 2 \\
\hline Sample size & 50 & 101 \\
\hline Mean & 7.82 & 0.24 \\
\hline Lower quartile & 1.39 & 1.45 \\
\hline Upper quartile & 10.04 & 0.80 \\
\hline Median & 5.32 & 1.25 \\
\hline Variance & 84.75 & 14.41 \\
\hline Kurtosis & 7.23 & 3.05 \\
\hline
\end{tabular}




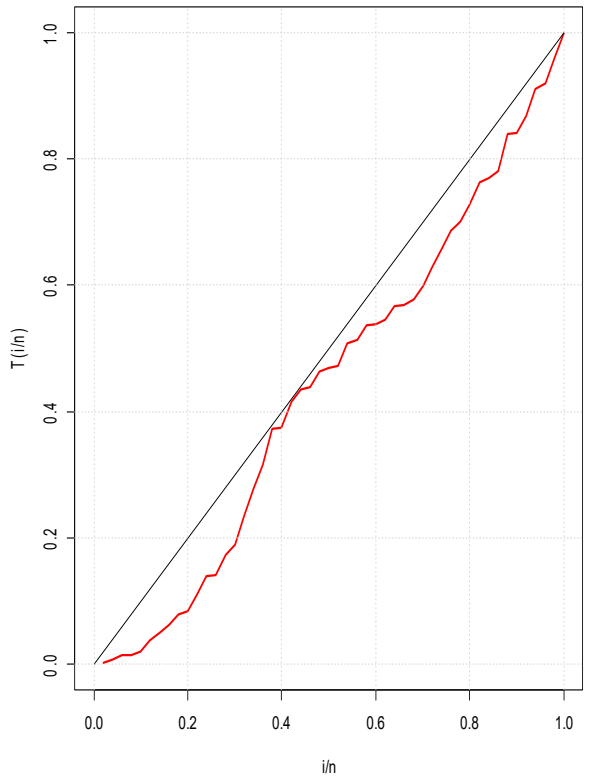

Diagram I

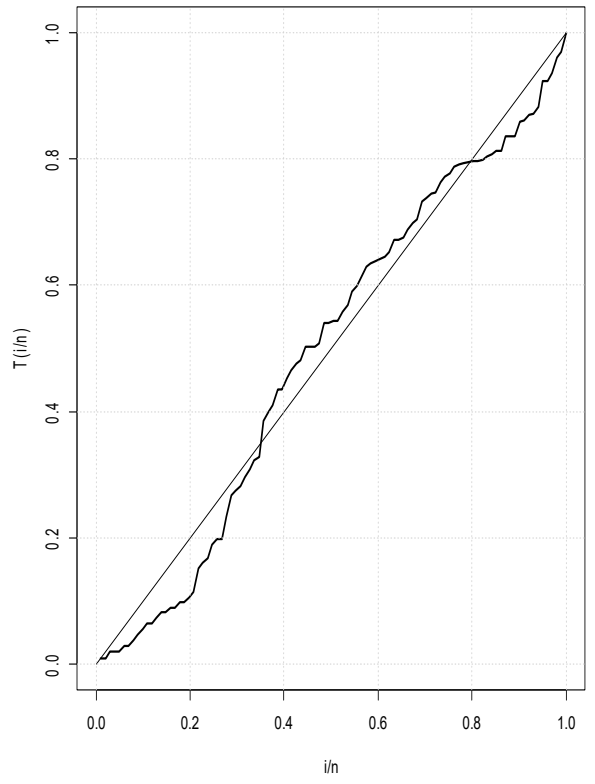

Diagram 2

Figure 5. TTT Plot for the two failure data

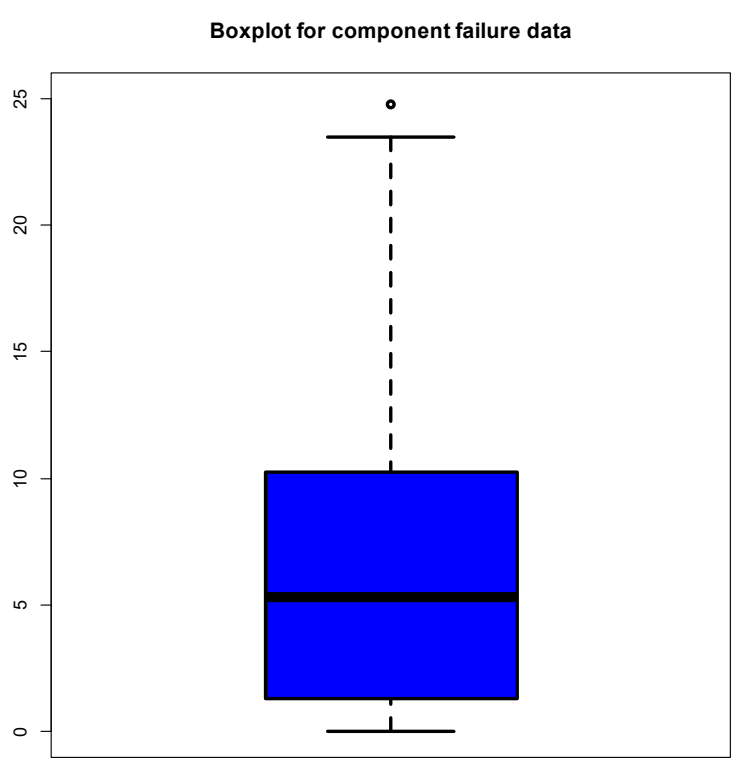

Diagram I

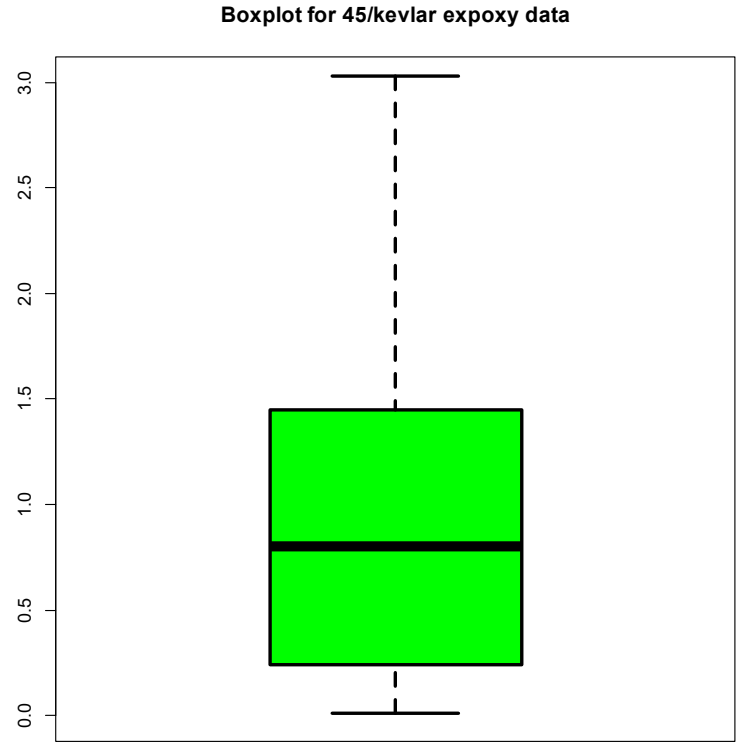


Poisson inverse exponential distribution, Alpha Power Extended Inverse Weibull distribution, and Alpha Power Extended Inverse Exponential distribution, with the following PDFs:

$$
\begin{array}{ll}
f(x)=\frac{\eta \lambda x^{-2} \log \alpha e^{-\eta x^{-1}} \alpha^{e^{-\eta x^{-1}}} \exp \left[-\lambda\left(\frac{\alpha^{e^{-\eta x^{-1}}}-1}{\alpha-1}\right)\right]}{(\alpha-1)\left(e^{-\lambda}-1\right)}, x>0 ; \alpha, \eta, \lambda>0 \\
f(x)=\frac{\eta \omega x^{-\omega-1} \log \alpha e^{-\eta x^{-\omega}} \alpha^{e^{-\eta x^{-\omega}}}}{(\alpha-1)}, & x>0 ; \alpha, \eta, \omega>0 \\
f(x)=\frac{\eta x^{-2} \log \alpha e^{-\eta x^{-1}} \alpha^{e^{-\eta x^{-1}}}}{(\alpha-1)}, & x>0 ; \alpha, \eta>0
\end{array}
$$

\begin{tabular}{|c|c|c|c|c|c|c|c|c|}
\hline Model & $\alpha$ & $\lambda$ & $\eta$ & $\omega$ & $-2 l$ & $A I C$ & $B I C$ & $K$ \\
\hline$A P E I W P$ & $\begin{array}{c}0.07 \\
(0.08) \\
\{-0.09,0.22\} \\
\end{array}$ & $\begin{array}{c}8.36 \\
(2.93) \\
\{2.62,14.10\} \\
\end{array}$ & $\begin{array}{c}2.94 \\
(0.39) \\
\{2.18,3.70\}\end{array}$ & $\begin{array}{c}0.23 \\
(0.03) \\
\{0.17,0.29\}\end{array}$ & 232.06 & 240.07 & 250.53 & 0.11 \\
\hline APPIE & $\begin{array}{c}6.91 \\
(3.618) \\
\{-0.19,14.01\} \\
\end{array}$ & $\begin{array}{c}-3.39 \\
(0.763) \\
\{-4.87,-1.91\}\end{array}$ & $\begin{array}{c}0.048 \\
(0.011) \\
\{0.03,0.07\} \\
\end{array}$ & $\begin{array}{l}- \\
(-) \\
\{-\}\end{array}$ & 247.40 & 253.41 & 261.25 & 0.18 \\
\hline APEIW & $\begin{array}{c}0.02 \\
(0.01) \\
\{0.00,0.04\}\end{array}$ & $\begin{array}{l}- \\
(-) \\
\{-\}\end{array}$ & $\begin{array}{c}1.64 \\
(0.30) \\
\{1.05,2.23\}\end{array}$ & $\begin{array}{c}0.32 \\
(0.04) \\
\{0.24,0.40\}\end{array}$ & 247.32 & 253.31 & 261.16 & 0.19 \\
\hline APEIE & $\begin{array}{c}8.21 \\
(2.34) \\
\{3.62 .12 .77\} \\
\end{array}$ & $\begin{array}{l}- \\
(-) \\
\{-\}\end{array}$ & $\begin{array}{c}0.10 \\
(0.01) \\
\{0.08,0.12\}\end{array}$ & $\begin{array}{l}- \\
(-) \\
\{-\}\end{array}$ & 279.40 & 283.40 & 288.63 & 0.32 \\
\hline$I W$ & $\begin{array}{l}- \\
(-) \\
\{-\}\end{array}$ & $\begin{array}{l}- \\
(-) \\
\{-\}\end{array}$ & $\begin{array}{c}0.42 \\
(0.06) \\
\{0.30,0.54\}\end{array}$ & $\begin{array}{c}0.62 \\
(0.04) \\
\{0.54,0.70\}\end{array}$ & 264.88 & 268.88 & 274.11 & 0.19 \\
\hline
\end{tabular}

Table 4. Analytical results of the APEIWP model and other competing models for Kevlar 45/epoxy data

Table 5. Analytical results of the APEIWP model and other competing models for failure time of components

\begin{tabular}{|c|c|c|c|c|c|c|c|c|}
\hline Model & $\alpha$ & $\lambda$ & $\eta$ & $\omega$ & $-2 l$ & $A I C$ & $B I C$ & $K$ \\
\hline APEIP & 7.81 & -3.82 & 0.20 & 0.69 & 323.00 & 331.01 & 338.65 & 0.17 \\
& $(3.86)$ & $(1.43)$ & $(0.08)$ & $(0.07)$ & & & \\
& $\{0.24,15.38\}$ & $\{-6.62,-1.02\}$ & $\{0.04,0.36\}$ & $\{0.55,0.83\}$ & & & \\
\hline APPIE & 10.76 & -4.99 & 0.13 & - & 343.42 & 349.41 & 355.15 & 0.30 \\
& $(5.73)$ & $(1.09)$ & $(0.03)$ & $(-)$ & & & \\
& $\{-0.47,21.99\}$ & $\{-7.13,-2.85\}$ & $\{0.07,0.19\}$ & $\{-\}$ & & & \\
\hline APEIW & 8.40 & - & 0.59 & 0.61 & 330.54 & 336.55 & 342.28 & 0.21 \\
& $(5.35)$ & $(-)$ & $(0.13)$ & $(0.05)$ & & & \\
& $\{-2.09,18.89\}$ & $\{-\}$ & $\{0.34,0.85\}$ & $\{0.51,0.71\}$ & & & \\
\hline APEIE & 19.27 & - & 0.27 & $(0.04)$ & $(-)$ & & & \\
& $(6.98)$ & $(-)$ & $\{-\}$ & $\{0.19,0.35\}$ & $\{-\}$ & & & \\
& $\{5.59,32.95\}$ & - & 1.117 & 0.48 & 337.28 & 341.28 & 345.11 & 0.20 \\
& $(-)$ & $(-)$ & $(0.17)$ & $(0.05)$ & & & \\
\hline
\end{tabular}

Based on Tables 4 and 5, it is evident that APEIWP model provides the best fit among other competing models, and can therefore be taken as the best model based on the data considered. Also, Figures 7 and 8 provide more information on the applicability of the APEIWP distribution in modeling lifetime data. 

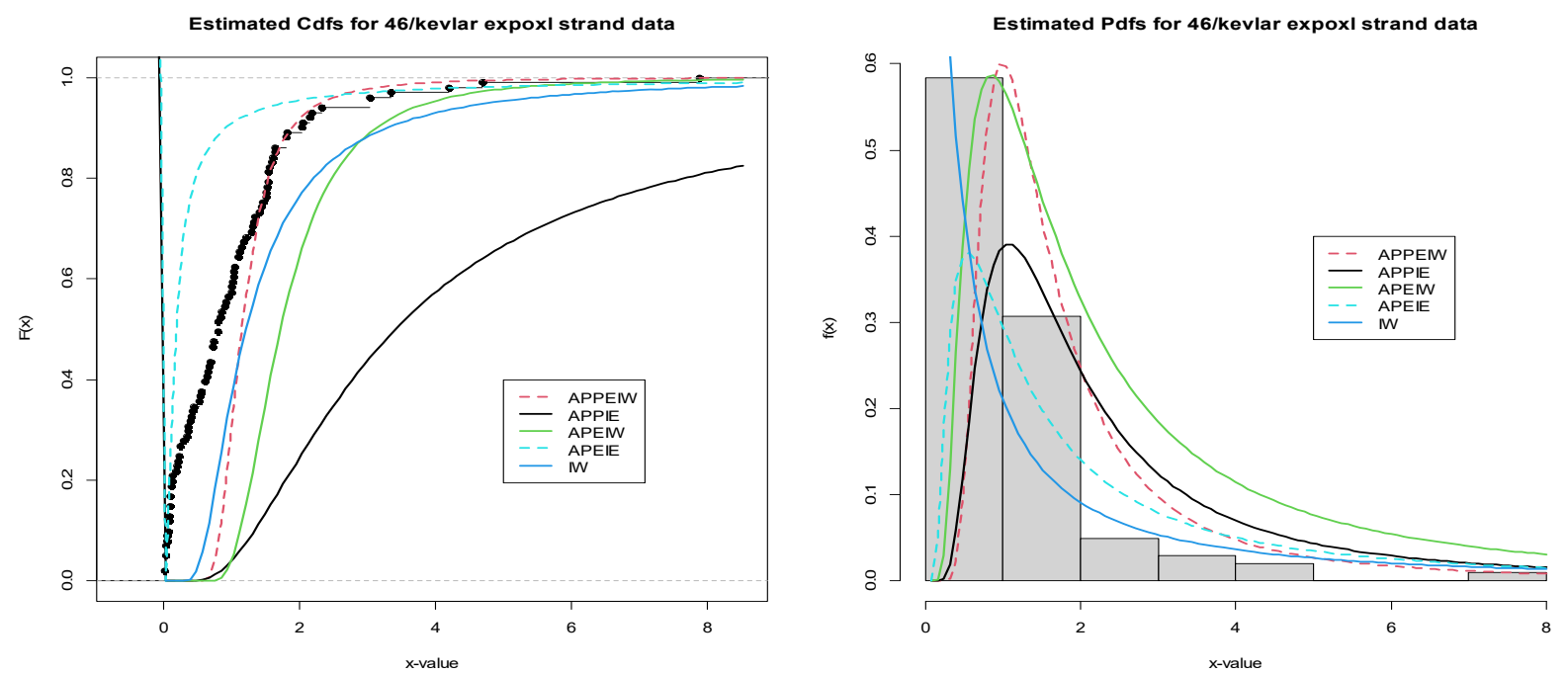

Figure 7. Estimated CDF and PDF function and other competing models for 46/Kevlar epoxy strand data
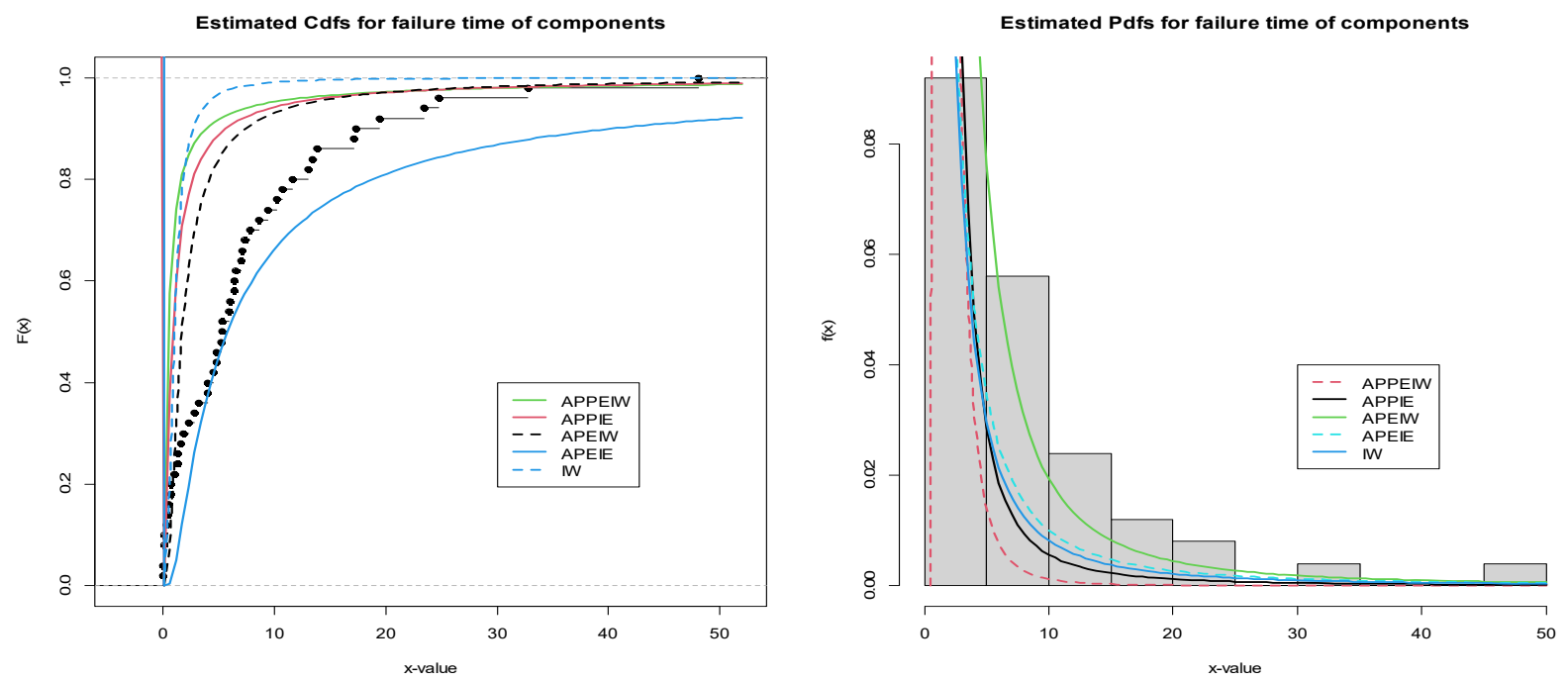

Figure 8. Estimated CDF and PDF function and other competing models for components failure data

\section{Conclusion}

In this work, we study the Alpha power extended inverse Weibull Poisson distribution. Some structural properties of the APEIWP distribution are derived such as ordinary and incomplete moments, Renyi entropy, order statistics, mean residual life, mean inactivity time, Bonferroni and Lorenz curves, and stress strength reliability. Estimation of the population parameters is carried out by using the maximum likelihood estimation method. Simulation study and two life data sets are used to illustrate the applicability of APEIWP distribution in modeling lifetime data. We recommend that further studies should be carried out by using different estimations techniques such as the Weighted Least Square method, Minimum spacing method, and Bayesian method, etc., and compare the performance of the estimation techniques.

\section{References}

Afify, A. Z., Alizadeh, M., Yousof, H. M., Aryal, G., \& Ahmad, M. (2016). The transmuted geometric-G family of distributions: theory and applications. Pakistan Journal of Statistics, 32(2), 139-160.

Afify, A. Z., Cordeiro, G. M., Yousof, H. M. Alzaatreh, A., and Nofal, Z. M. (2016b). The Kumaraswamy transmuted-G family of distributions: properties and applications. Journal of Data Science, 14, 245-270. https://doi.org/10.6339/JDS.201604_14(2).0004 
Alizadeh, M., Ghosh, I., Yousof, H. M., Rasekhi, M., \& Hamedani G. G. (2017). The generalized odd generalized exponential family of distributions: properties, characterizations, and applications. Journal of Data Science, 15, 443466. https://doi.org/10.6339/JDS.201707_15(3).0005

Alizadeh, M., Rasekhi, M., Yousof, H. M., \& Hamedani G. G. (2018). The transmuted Weibull G family of distributions. Hacettepe Journal of Mathematics and Statistics, 47, 1-20. https://doi.org/10.15672/HJMS.2017.440

Andrews, D. F., \& Herzberg. A. M. (2012). Data: a collection of problems from many fields for the student and research worker. Springer Science and Business Media.

Aryal, G. R., \& Yousof, H. M. (2017). The exponentiated generalized-G Poisson family of distributions. Economic Quality Control, 32, 1-17. https://doi.org/10.1515/eqc-2017-0004

Aryal, G., \& Elbatal, I. (2015). Kumaraswamy modified inverse Weibull distribution: Theory and application. Applied Mathematics \& Information Sciences, 9(2), 651.

Azzalini, A. (1985). A class of distributions that includes the normal ones. Scandinavian journal of statistics. pages $171-178$.

Basheer, A. M. (2019). Alpha power inverse Weibull distribution with reliability application. Journal of Taibah University for Science, 13(1), 423-432. https://doi.org/10.1080/16583655.2019.1588488

Bourguignon, M., Silva, R. S., \& Cordeiro, G. M. (2014). The Weibull-G Family of Distributions. Journal of Data Science, 12, 53-68. https://doi.org/10.6339/JDS.201401_12(1).0004

Cordeiro, G. M., \& de Castro, M. (2011). A new family of generalized distributions. Journal of Statistical Computation and Simulation. 81, 883-898. https://doi.org/10.1080/00949650903530745

Cordeiro, G. M., Afify, A. Z., Ortega, E. M. M., Suzuki, A. K., \& Mead, M. E. (2019). The odd Lomax generator of distributions: properties, estimation, and applications. Journal of Computational and Applied Mathematics, 347, 222-237. https://doi.org/10.1016/j.cam.2018.08.008

Cordeiro, G. M., Afify, A. Z., Yousof, H. M., Pescim, R. R., \& Aryal, G. R. (2017). The exponentiated Weibull family of distributions: theory and applications. Mediterranean Journal of Mathematics, 14, 1-22. https://doi.org/10.1007/s00009-017-0955-1

De Gusmao, F. R., Ortega, E. M., \& Cordeiro, G. M. (2011). The generalized inverse Weibull distribution. Statistical Papers, 52(3), 591-619. https://doi.org/10.1007/s00362-009-0271-3

Dey, S., Alzaatreh, A., Zhang, C., \& Kumar, D. (2017a). A New Extension of Generalized Exponential Distribution with Application to Ozone Data. Science and Engineering. https://doi.org/10.1080/01919512.2017.1308817

Dey, S., Ghosh, I., \& Kumar, D. (2019). Alpha-power transformed Lindley distribution: properties and associated inference with application to earthquake data. Annals of Data Science, 6(4), 623-650. https://doi.org/10.1007/s40745-018-0163-2

Dey, S., Nassar, M., \& Kumar, D. (2018). Alpha power transformed inverse Lindley distribution: A distribution with an upside-down bathtub-shaped hazard function. Journal of Computational and Applied Mathematics, 348, 130-145. https://doi.org/10.1016/j.cam.2018.03.037

Dey, S., Sharma, V. K., \& Mesfioui, M. (2017b). A New Extension of Weibull Distribution with Application to Lifetime Data. Annals of Data. Science. https://doi.org/10.1007/s40745-016-0094-8

Eugene, N., Lee, C., \& Famoye, F. (2002). Beta-normal distribution and its applications. Communications in Statistics-Theory and methods, 31(4), 497-512. https://doi.org/10.1081/STA-120003130.

Hassan, A. S, Mohammed, R. E., Elgarhy, M., \& Fayomi, A. (2018). Alpha power transformed extended exponential distribution: properties and applications. Journal of Nonlinear Sciences and Applications, 12(4), 62-67. https://doi.org/10.22436/jnsa.012.04.05

Hassan, A. S., \& Elgarhy, M. (2016). Kumaraswamy Weibull-generated family of distributions with applications. Advances and Applications in Statistics, 48, 205-239. https://doi.org/10.17654/AS048030205

Hassan, A. S., Marwa, A. A., Zaher, H., \& Elsherpieny, E. A. (2014). Comparison of Estimators for Exponentiated Inverted Weibull Distribution Based on Grouped Data. International Journal of Engineering Research and Application, 4(1), 77-90.

Keller, A. Z., Kamath, A. R. R., \& Perera, U. D. (1982). Reliability analysis of C N C machine tools. Reliability Engineering, 3(6), 449-473. https://doi.org/10.1016/0143-8174(82)90036-1 
Khan, M. S. (2010). The beta inverse Weibull distribution. International Transactions in Mathematical Sciences and Computer, 3(1), 113-119.

Khan, M. S., \& King, R. (2012). Modified inverse Weibull distribution. Journal of statistics applications \& Probability, 1(2), 115. https://doi.org/10.12785/jsap/010204

Korkmaz, M. C., Yousof, H. M., Hamedani, G. G., \& Ali, M. M. (2018). The Marshall-Olkin generalized-G Poisson family of distributions, Pakistan Journal of Statistics, 34(3), 251-267.

Mahdavi, A., \& Kundu, D. A. (2017). A new method for generating distributions with an application to the exponential distribution. Communications in Statistics-Theory and Methods, 46(13), 6543-57. https://doi.org/10.1080/03610926.2015.1130839

Marshall, A. W., \& Olkin, I. (1997). A new method for adding a parameter to a family of distributions with application to the exponential and Weibull families. Biometrica, 84(3), 641-652. https://doi.org/10.1093/biomet/84.3.641

Moors, J. J. A. (1998). A quantile alternative for kurtosis. Journal of the Royal Statistical Society Ser. D., The Statistician, 37, 25-32. https://doi.org/10.2307/2348376

Mudholkar, G. S., \& Srivastava, D. K. (1993). Exponentiated Weibull family for analyzing bathtub failure-rate data. IEEE Transactions on Reliability. 42(2), 299-302. https://doi.org/10.1109/24.229504

Murthy, D. N. P., Xi, M., \& Jiangs, R. (2004). Weibull model. Wiley, Hoboken.

Nassar, M., Alzaatreh, A., Mead, M., \& Abo-Kasem, O. (2017). Alpha power Weibull distribution: Properties and applications. Communications in Statistics-Theory and Methods, 46(20), 10236-52. https://doi.org/10.1080/03610926.2016.1231816

Ogunde, A. A., Fayose, S. T., Ajayi, B., \& Omosigho, D. O. (2020). Properties, Inference, and Applications of Alpha Power Extended Inverted Weibull Distribution. International Journal of Statistics and Probability, 9(6). https://doi.org/10.5539/ijsp.v9n6p90

Okasha, H. M., El-Baz, A. H., Tarabia, A. M. K., \& Basheer, A. M. (2017). Extended inverse Weibull distribution with reliability application. Journal of the Egyptian Mathematical Society, 25(3), 343-349. https://doi.org/10.1016/j.joems.2017.02.006

Oluyede, B. O., \& Yang, T. (2014). Generalizations of the inverse Weibull and related distributions with applications," Electronic Journal of Applied Statistical Analysis, 7, 94- 116.

Oluyede, B. O., Pu, S., Makubate, B., \& Qiu, Y. (2018). The Gamma Weibull-G Family of Distributions. Austrian Journal of Statistics, 47(1), 45-76. https://doi.org/10.17713/ajs.v47i1.155

Pararai, M., Warahena-Liyanage, G., \& Oluyede, B. O. (2014). A new class of generalized inverse Weibull distribution with applications. Journal of Applied Mathematics and Bioinformatics, 4, 17-35.

Said, A., Ahmed, Z., Afify, I., Elbatal, \& Elgarhy, M. (2020). The Extended Inverse Weibull Distribution: Properties and Applications. Hindawi Complexity. Article ID 3297693. https://doi.org/10.1155/2020/3297693

Yousof, H. M., Afify, A. Z., Alizadeh, M., Butt, N. S., Hamedani, G. G., \& Ali, M. M. (2015). The transmuted exponentiated generalized-G family of distributions. Pakistan Journal Statistics and Operation Research, 11, 441-464. https://doi.org/10.18187/pjsor.v11i4.1164

\section{Copyrights}

Copyright for this article is retained by the author(s), with first publication rights granted to the journal.

This is an open-access article distributed under the terms and conditions of the Creative Commons Attribution license (http://creativecommons.org/licenses/by/4.0/). 